\title{
Increased epithelial permeability in pulmonary fibrosis in relation to disease progression
}

\author{
N.S.L. Goh*, S.R. Desai", C. Anagnostopoulos ${ }^{*}$, D.M. Hansell*, R.K. Hoyles*, \\ H. Sato*, C.P. Denton ${ }^{+}$, C.M. Black ${ }^{+}$, R.M. du Bois ${ }^{\S}$ and A.U. Wells*
}

ABSTRACT: Epithelial injury contributes to pathogenesis in idiopathic pulmonary fibrosis (IPF) but its role in the interstitial lung disease (ILD) of systemic sclerosis (SSc) is uncertain. We quantified the prognostic significance of inhaled technetium-99m $\left({ }^{99 m} \mathrm{Tc}\right)$-labelled diethylene triamine pentacetate (DTPA) pulmonary clearance, a marker of the extent of epithelial injury, in both diseases.

Baseline ${ }^{99 m}$ Tc-DTPA pulmonary clearance was evaluated retrospectively in patients with SScILD $(n=168)$ and IPF $(n=97)$ against mortality and disease progression.

In SSc-ILD, the rapidity of total clearance (hazard ratio (HR) $1.02,95 \% \mathrm{Cl} 1.01-1.03 ; p=0.001$ ) and the presence of abnormally rapid clearance (HR 2.10; 95\% Cl 1.25-3.53; $p=0.005$ ) predicted a shorter time to forced vital capcity (FVC) decline, independent of disease severity. These associations were robust in both mild and severe disease. By contrast, in IPF, delayed clearance of the slow component, an expected consequence of honeycomb change, was an independent predictor of a shorter time to FVC decline (HR 1.01, 95\% Cl 1.00-1.02; $p<0.01$ ).

Epithelial injury should be incorporated in pathogenetic models in SSc-ILD. By contrast, outcome is not linked to the overall extent of epithelial injury in IPF, apart from abnormalities ascribable to honeycombing, suggesting that core pathogenetic events may be more spatially focal in that disease.

KEYWORDS: Diethylene tiamine pentacetate clearance, epithelial permeability, fibrosis, pathogenetic, progression

$\mathrm{n}$ the interstitial lung disease (ILD) of systemic sclerosis (SSc), epithelial injury has been linked to the presence and progression of lung disease [1-3], but a pathogenetic contribution from epithelial injury has yet to be established. By contrast, in idiopathic pulmonary fibrosis (IPF), there is substantial evidence that epithelial cell dysfunction contributes to the fibrotic microenvironment $[4,5]$, and markers of epithelial injury have been linked to outcome [1, 6-8]. Clearance of inhaled technetium-99m $\left({ }^{99 \mathrm{~m}} \mathrm{Tc}\right)$-labelled diethylene triamine pentacetate (DTPA) from the lung, a measure of epithelial permeability/injury [9], is abnormal in several interstitial lung diseases, including SSc-ILD [1, $10]$ and IPF $[1,8,11]$.

However, the possibility exists, with any prognostic marker, that relationships to a poor outcome might merely represent associations with more advanced disease. In a study of patients with SSc-ILD or idiopathic interstitial pneumonia, WeLLS et al. [1] reported that rapid total ${ }^{99 \mathrm{~m}}$ Tc-DTPA clearance was associated with disease progression, but no adjustment was made for disease severity in that analysis. More rapid ${ }^{99 \mathrm{~m}}$ Tc-DTPA clearance has been associated with lower diffusing capcity of the lung for carbon monoxide (DL,CO) levels in SSc [12] and with more extensive disease on high-resolution computed tomography (HRCT) in IPF [11]. In IPF, a reported relationship between rapid clearance in the fast component of ${ }^{99 \mathrm{~m}} \mathrm{Tc}$-DTPA clearance and mortality was substantially weaker than the relationship between mortality and lower DL,CO [8].

Therefore, the aim of the present study was to examine the relationships between the rapidity of ${ }^{99 \mathrm{~m}} \mathrm{Tc}$-DTPA clearance and outcome in IPF and SSc-ILD, with and without adjustment for disease

\section{AFFILIATIONS}

${ }^{*}$ Royal Brompton Hospital and National Heart and Lung Institute, \#King's College Hospital,

"Barts and The London School of Medicine and Dentistry, and ${ }^{+}$Royal Free Hospital, London, UK. ${ }^{\text {s}}$ National Jewish Health, Denver, CO, USA.

\section{CORRESPONDENCE}

A.U. Wells

Interstitial Lung Disease Unit, Royal Brompton Hospital and NHLI

Imperial College

Emmanuel Kaye Building

1B Manresa Road

London SW3 6LP UK

E-mail: athol.wells@rbht.nhs.uk

Received:

Jan 212010

Accepted after revision:

Sept 232010

First published online:

Oct 282010 
severity (as judged by HRCT and pulmonary function tests (PFTs)).

\section{METHODS \\ Patients}

Patients in both disease cohorts have been included in other published studies of outcome [13-15].

\section{SSC-ILD}

The SSc-ILD cohort comprised 168 out of 277 consecutively referred patients with SSc-ILD on HRCT (January 1990December 1999). Exclusion criteria are listed in the online supplementary material. Serial PFTs were available in 152 out of $168(90 \%)$ patients.

\section{IPF}

The IPF cohort comprised 97 out of 191 consecutively referred patients meeting American Thoracic Society (ATS) criteria for IPF (December 1990-December 1996) [16]. Exclusion criteria are listed in the online supplementary material. Time-todecline analyses were performed in 89 out of 97 patients (serial PFTs, $n=73$; death within 6 months of presentation, $n=16$ ).

\section{Clinical data}

Vital status (on May 1, 2006) and serial PFTs (up to May 1, 2006) were recorded. Patients were categorised as former smokers ( $>1$ cigarette $\cdot \mathrm{day}^{-1}$ for $>1 \mathrm{yr}$ ) or lifelong nonsmokers. Treatment was defined as corticosteroid and/or immunosuppressive (cyclophosphamide, azathioprine or mycophenolate mofetil) therapy at the time of ${ }^{99 \mathrm{~m}} \mathrm{Tc}$-DTPA clearance (see online supplementary material for more details). Therapeutic status was defined as "intention to treat" (treatment from presentation or within 3 months of presentation) or "intention to observe" (no treatment within 3 months of presentation).

PFTs (expressed as \% predicted) and HRCT were performed as previously reported (online supplementary material) [14]. The composite physiologic index (CPI) [17] was calculated as follows.

$$
\begin{aligned}
\mathrm{CPI}= & 91.0-(0.65 \times D \mathrm{~L}, \mathrm{CO} \% \text { pred })-(0.53 \times \mathrm{FVC} \\
& \% \text { pred })+(0.34 \times \mathrm{FEV} 1 \% \text { pred })
\end{aligned}
$$

where FVC is forced vital capacity and FEV1 is forced expiratory volume in $1 \mathrm{~s}$. All investigations were performed as part of a prospective routine clinical protocol.

\section{Staging status in SSC-ILD}

Disease severity was quantified as "mild" (HRCT disease extent $\leqslant 10 \%$ or FVC $\geqslant 70 \%$ pred in "indeterminate" disease (HRCT disease extent 10-30\%)) or "extensive" (HRCT disease extent $>30 \%$ or FVC $<70 \%$ pred in indeterminate disease) [14].

\section{${ }^{99 m}$ Tc-DTPA pulmonary clearance}

Lung clearance of aerosolised ${ }^{99 \mathrm{~m}} \mathrm{Tc}-\mathrm{DTPA}$ was measured as: 1) total clearance; 2 ) normal versus abnormally rapid clearance; and 3) the fast and slow components of clearance in the majority of patients with recorded raw data (online supplementary material) $[18,19]$.

\section{Outcome}

Mortality and disease progression (time to decline in pulmonary function indices) were quantified from the date of
${ }^{99 m}$ Tc-DTPA clearance. Significant changes in PFTs, using ATS and European Respiratory Society criteria [16], were defined as 1) $\geqslant 10 \%$ decline in FVC and 2$) \geqslant 15 \%$ decline in $\mathrm{DL}, \mathrm{CO}$ on two consecutive occasions. When follow-up ended with functional deterioration on a single occasion, this was taken to indicate significant decline, with the proviso that there was symptomatic or radiographic evidence of deterioration. "Progression-free survival", defined as the time to a progression event (decline in FVC or DL,CO, or death) [20], was also evaluated.

The rate of change in FVC and DL,CO levels (in millilitres per year), quantified as the difference between the last and first measurement over the total lung function follow up time, was also examined in relation to the speed of total DTPA clearance.

\section{Data analysis}

Analyses were performed using STATA software (StataCorp, College Station, TX, USA). Data are presented as mean \pm SD or median (range), depending on distribution. Group comparisons were made using unpaired t-tests, Wilcoxon rank sum, Chi-squared statistics and Fisher's exact test, as appropriate.

${ }^{99} \mathrm{~m}$ Tc-DTPA clearance variables were examined in separate models against mortality and time to disease progression using proportional hazards analysis (online supplementary material). Variables not contributing to equation explanatory power ( $p>0.05)$ were excluded, using a stepwise method.

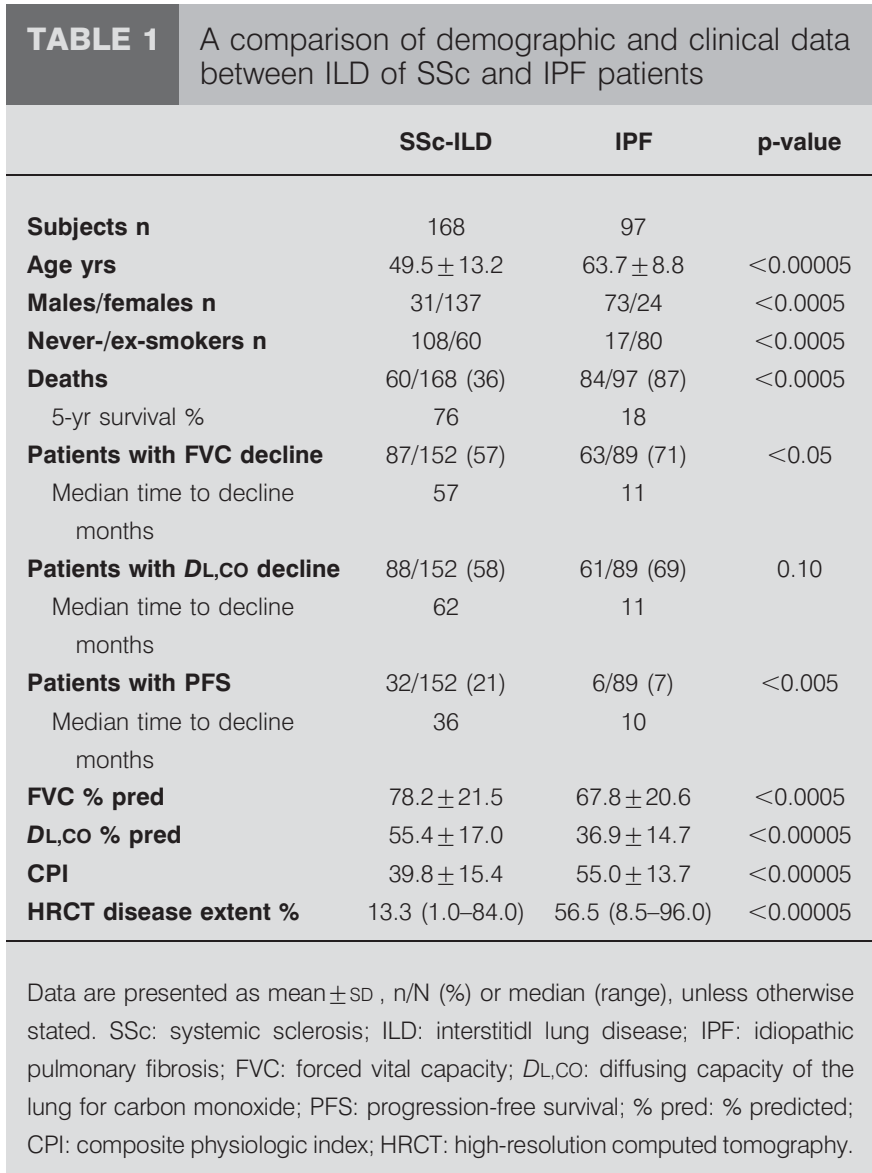




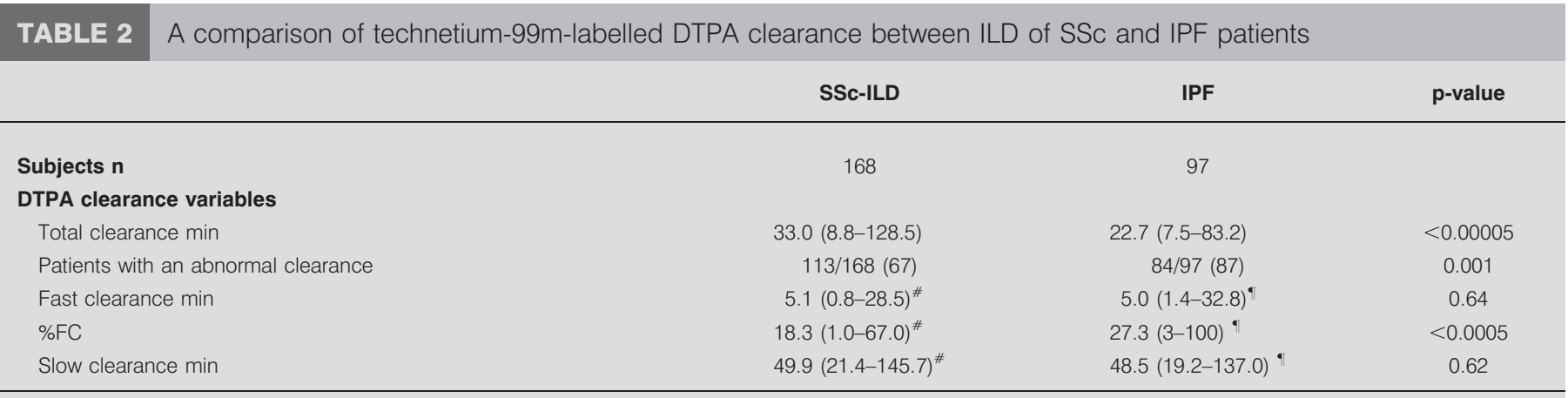

Data are presented as median (range) or n/N (\%), unless otherwise stated. SSc: systemic sclerosis; ILD: interstitial lung disease; IPF: idiopathic pulmonary fibrosis; DTPA: diethylene triamine pentacetate; \%FC: \% DTPA cleared by the fast component. ${ }^{\#}: \mathrm{n}=126 ;{ }^{\bullet}: \mathrm{n}=81$.

\section{RESULTS}

As shown in table 1, when compared with SSc-ILD patients, IPF patients were older, had more severe disease at presentation and exhibited a worse prognosis. IPF patients had markedly faster total clearance $(\mathrm{p}<0.00005)$ and a higher proportion of patients with abnormal total clearance $(p=0.001)$ (table 2$)$.

\section{${ }^{99 m}$ Tc-DTPA clearance in relation to disease severity}

In SSc-ILD (online supplementary table E1), clearance was increasingly rapid in increasingly severe disease (CPI Spearman's rho $(\mathrm{rs})=-0.37(\mathrm{p}<0.00005$; online supplementary fig. E1) versus HRCT disease extent $r_{s}=-0.31(p=0.0001$; online supplementary fig. E2)). \% DTPA cleared by the fast component $(\% \mathrm{FC})$ was also associated with both measures of disease severity (CPI rs $=0.42(\mathrm{p}<0.00005)$ versus HRCT disease extent $\left.r_{s}=0.25(p=0.005)\right)$.

In IPF (online supplementary table E2), only \%FC was associated with the CPI ( $\left.\mathrm{r}_{\mathrm{s}}=0.22 ; \mathrm{p}=0.05\right)$ and HRCT extent $(\mathrm{r}=0.24 ; \mathrm{p}=0.03)$.

\section{Prognostic value of ${ }^{99 m}$ TC-DTPA clearance in SSc-ILD}

Mortality

Mortality was associated with increasingly rapid total clearance (hazard ratio (HR) 1.02, 95\% CI 1.00-1.03; p=0.02) (table 3 ) but this association was lost $(\mathrm{p}>0.10)$ with adjustment for age, sex, smoking status and disease severity (CPI levels and staging status in alternative models). Mortality was not linked to the presence of abnormally rapid clearance.

Time to decline in pulmonary function variables

A shorter time to FVC decline was predicted by increasing rapid total clearance (HR 1.02, 95\% CI 1.01-1.03; $\mathrm{p}=0.001$ ) and the presence of abnormally rapid clearance (HR 2.10, 95\% CI 1.25-3.53; $p=0.005$ ) (table 4). These variables were the only independent predictors of a shorter time to FVC decline in separate multivariate models (whether disease severity was quantified using CPI levels or staging status). By contrast, time to $D \mathrm{~L}, \mathrm{CO}$ decline was predicted by neither the rapidity of total clearance nor the presence of abnormally rapid clearance (table 5).

TABLE 3 A comparison of mortality and progression-free survival in relation to technetium-99m-labelled DTPA clearance in ILD of SSc and IPF patients

\begin{tabular}{|c|c|c|c|c|c|c|c|c|}
\hline \multirow[t]{3}{*}{ DTPA clearance variable } & \multicolumn{4}{|c|}{ Mortality } & \multicolumn{4}{|c|}{ Progressive-free survival } \\
\hline & \multicolumn{2}{|c|}{ SSc-ILD } & \multicolumn{2}{|c|}{ IPF } & \multicolumn{2}{|c|}{ SSc-ILD } & \multicolumn{2}{|l|}{ IPF } \\
\hline & HR (95\% Cl) & p-value & HR $(95 \% \mathrm{Cl})$ & p-value & HR $(95 \% \mathrm{Cl})$ & p-value & $\mathrm{HR}(95 \% \mathrm{Cl})$ & p-value \\
\hline $\begin{array}{l}\text { Increasing rapid total } \\
\text { clearance }^{\#} \text { min }\end{array}$ & $1.02(1.00-1.03)$ & 0.02 & $1.01(0.99-1.02)$ & 0.28 & $1.01(1.00-1.02)$ & 0.006 & $1.00(0.98-1.01)$ & 0.58 \\
\hline $\begin{array}{l}\text { Patients with an abnormal } \\
\text { clearance }^{\#} \text { min }\end{array}$ & $1.21(0.69-2.12)$ & 0.51 & $1.56(0.82-2.96)$ & 0.18 & $1.59(1.06-2.38)$ & 0.02 & $0.93(0.49-1.77)$ & 0.83 \\
\hline $\begin{array}{l}\text { Increasing rapid fast } \\
\text { clearance } \mathrm{min}\end{array}$ & $1.01(0.91-1.13)$ & 0.81 & $1.01(0.96-1.07)$ & 0.71 & $1.10(1.01-1.19)$ & 0.03 & $1.00(0.94-1.06)$ & 0.95 \\
\hline Increasing \%FC & $1.02(1.00-1.04)$ & 0.06 & $1.00(0.99-1.02)$ & 0.66 & $1.01(0.99-1.02)$ & 0.30 & $1.00(0.98-1.01)$ & 0.78 \\
\hline $\begin{array}{l}\text { Increasing delayed slow } \\
\text { clearance } \min \end{array}$ & $1.00(0.98-1.01)$ & 0.60 & $1.00(0.99-1.01)$ & 0.38 & $1.00(0.99-1.01)$ & 0.61 & $1.01(1.00-1.02)$ & 0.01 \\
\hline
\end{tabular}

DTPA: diethylene triamine pentacetate; ILD: interstitial lung disease; SSc: systemic sclerosis; IPF: idiopathic pulmonary fibrosis; HR: hazard ratio; \%FC: \% DTPA cleared by the fast component. ${ }^{*}: 168$ SSc-ILD patients and 97 IPF patients; ${ }^{\prime}: 126$ SSc-ILD patients and 81 IPF patients. 


\begin{tabular}{|c|c|c|c|c|c|}
\hline TABLE 4 & \multicolumn{5}{|c|}{$\begin{array}{l}\text { A comparison of time to decline in FVC in relation to technetium-99m-labelled DTPA clearance in ILD of SSc and } \\
\text { IPF patients }\end{array}$} \\
\hline \multirow{3}{*}{\multicolumn{2}{|c|}{ DTPA clearance variable }} & \multicolumn{4}{|c|}{ Time to decline in FVC } \\
\hline & & \multicolumn{2}{|c|}{ SSC-ILD } & \multicolumn{2}{|c|}{ IPF } \\
\hline & & HR (95\% Cl) & $\mathrm{p}$-value & HR $(95 \% \mathrm{Cl})$ & p-value \\
\hline \multicolumn{2}{|c|}{ Patients with an abnormal clearance ${ }^{\#}$ min } & $2.10(1.25-3.53)$ & 0.005 & $0.59(0.28-1.26)$ & 0.18 \\
\hline \multicolumn{2}{|c|}{ Increasing rapid fast clearance $\min$} & $1.15(1.04-1.28)$ & $<0.01$ & $0.98(0.92-1.04)$ & 0.47 \\
\hline \multicolumn{2}{|c|}{ Increasing \% FC } & $1.02(1.00-1.04)$ & 0.02 & $0.99(0.97-1.01)$ & 0.39 \\
\hline \multicolumn{2}{|c|}{ Increasing delayed slow clearance $\min$} & $0.99(0.98-1.01)$ & 0.36 & $1.01(1.00-1.02)$ & $<0.01$ \\
\hline
\end{tabular}

Shorter progression-free survival (table 3 ) was associated with increasing rapid total clearance $(p=0.006)$, which was the only independent predictor on multivariate analysis (with severity quantified using CPI levels and staging status in separate models). Shorter progression-free survival was also predicted by the presence of abnormally rapid clearance $(p=0.02)$, but this association was lost on multivariate analysis.

\section{Correlation between rate of change in PFTs and speed of DTPA} clearance

In SSc-ILD, the speed of total DTPA clearance correlated with the rate of FVC change $(r=0.17 ; p=0.03)$, but not with the rate of $D L, C O$ change $(r=0.08 ; p=0.33)$. In IPF, the speed of total DTPA clearance did not correlate with the rate of FVC change $(r=-0.09$; $\mathrm{p}=0.44)$, nor the rate of $D \mathrm{~L}, \mathrm{CO}$ change $(\mathrm{r}=-0.14 ; \mathrm{p}=0.21)$.

Prognostic value of ${ }^{99 \mathrm{~m}} \mathrm{Tc}$-DTPA clearance in mild and extensive disease

The prognostic value of ${ }^{99 \mathrm{~m}} \mathrm{Tc}-\mathrm{DTPA}$ clearance was reexamined separately in mild $(n=115)$ and extensive $(n=53)$ disease (online supplementary table E3). A shorter time to FVC decline was linked to increasingly rapid total clearance in both mild $(p=0.02)$ and $(p<0.05)$ extensive disease. A shorter time to FVC decline was associated with the presence of abnormally rapid clearance in mild $(\mathrm{p}<0.05)$ but not in extensive disease. Neither clearance variable was predictive of either mortality (online supplementary table E4) or time to DL,CO decline (online supplementary table E3).

${ }^{99 m}$ Tc-DTPA clearance versus time to decline in FVC: influence of therapeutic status

In 65 patients receiving therapy within 3 months of measurement of ${ }^{99 \mathrm{~m}} \mathrm{Tc}$-DTPA clearance (the "intention to treat" patient subgroup), time to FVC decline was not linked to the rapidity of clearance. By contrast, in the remaining 87 patients (the "intention to observe" patient subgroup), a shorter time to FVC decline was associated with both increasingly rapid clearance (HR 1.03, 95\% CI 1.01-1.05; $p=0.002$ ) and the presence of abnormally rapid clearance (HR 2.29, 95\% CI 1.17-4.49; $\mathrm{p}=0.02)$.

TABLE 5 A comparison of time to decline in DL,CO in relation to technetium-99m-labelled DTPA clearance in ILD of SSc and IPF patients

DTPA clearance variable

Time to decline in DL,Co

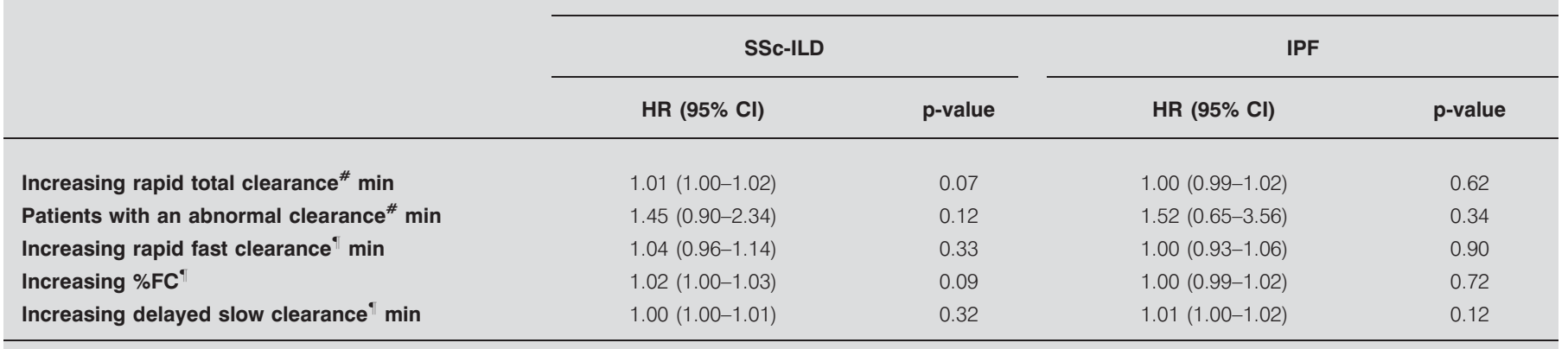

DL,CO: diffusing capacity of the lung for carbon monoxide; DTPA: diethylene triamine pentacetate; ILD: interstitial lung disease; SSc: systemic sclerosis; IPF: idiopathic pulmonary fibrosis; HR: hazard ratio; \%FC: \% DTPA cleared by the fast component. * : 168 SSc-ILD patients and 97 IPF patients; ${ }^{\prime}: 126$ SSc-ILD patients and 81 IPF patients. 


\section{Prognostic value of clearance curve components}

Outcome was re-examined against clearance curve components in 126 out of 168 patients (75\%). On univariate analysis, a shorter time to FVC decline was linked to increasingly rapid clearance of the fast component and a higher \%FC, but not the rapidity of clearance of the slow component (table 4). On multivariate analysis, a shorter time to FVC decline was linked to rapid clearance of the fast component and a higher \%FC (online supplementary material). Neither mortality (table 3) nor time to DL,CO decline (table 5) was linked to any clearance curve component.

Prognostic value of ${ }^{99 m}$ TC-DTPA clearance in IPF Neither mortality (table 3 ) nor progression of disease (tables 4 and 5) was linked to the rapidity of total clearance or the presence of abnormally rapid clearance. A shorter time to FVC decline (table 4) was associated with delayed clearance of the slow component $(p<0.01)$, but was not linked to the fast component or the \%FC. On multivariate analysis, the association between time to FVC decline and slow component clearance was independent of the extent of disease, as determined by HRCT or CPI, in separate models. Neither mortality nor time to $D \mathrm{~L}, \mathrm{CO}$ decline was linked to any clearance curve component.

\section{DISCUSSION}

Our findings indicate that in SSc-ILD, increased epithelial permeability is linked to disease severity and is predictive of disease progression, in both mild and extensive disease, and in the subset of patients not receiving immediate treatment. By contrast, increased epithelial permeability was not linked to disease severity in IPF and disease progression was associated only with a ${ }^{99 \mathrm{~m}}$ Tc-DTPA clearance profile indicative of abnormally slow clearance in some lung regions. These relationships persisted after adjustment for disease severity.

Although these observations might appear to suggest a clinical role for the measurement of ${ }^{99 \mathrm{~m}} \mathrm{Tc}$-DTPA clearance in SSc-ILD, the procedure is not without limitations, including confounding by current or recent smoking and radiation constraints, which also preclude quantification of the intrapatient variability of the test. Although routinely performed at our institution (Royal Brompton Hospital, London, UK), measurement of ${ }^{99 m}$ Tc-DTPA is not available in many centres. Thus, we have not explored the precision of prognostic evaluation, using receiver operating characteristic analysis, but have instead focussed on pathogenetic implications of our findings.

The findings in the present study provide support for a direct relationship between epithelial events and progression of fibrosis in SSc-ILD. Until now, the pathogenetic focus has been on vascular, immunological and fibrotic processes, with endothelial cell injury viewed by some as central to the pathogenesis of SSc vasculopathy [21]. However, indirect support for the pathogenetic importance of epithelial injury has been suggested in several studies. In SSc-ILD, early histological changes include both endothelial and epithelial injury, together with interstitial oedema and excess collagen deposition [22]. Epithelial cells may also contribute to the fibrotic process through epithelial-mesenchymal transition [23]. Rapid clearance of ${ }^{99 \mathrm{~m}} \mathrm{Tc}$-DTPA has been linked to functional decline in a small cohort that included patients with SSc-ILD [1]. However, in that study, patients with idiopathic interstitial pneumonia were also included and, crucially, disease severity was not taken into account. In the present study, all relationships between disease progression and increased epithelial permeability were independent of disease severity, as judged by functional and morphological quantification in separate models, and, thus, it seems unlikely that epithelial injury is linked to outcome in SSc-ILD only as an epiphenomenon. Furthermore, epithelial cells are a rich source of a number of profibrotic cytokines, including transforming growth factor (TGF)- $\beta 1$ [24, 25], platelet-derived growth factor [26] and tumour necrosis factor- $\alpha$ [24, 27]. More compellingly, in a recent transgenic mouse model of scleroderma [28], even minor epithelial injury was shown to induce significant pulmonary fibrosis, mediated by perturbation of TGF- $\beta$ signalling, supporting an important pathogenetic role for epithelial injury. Taken together, the current and previous observations suggest that epithelial events are likely to be pathogenetically important in SSc-ILD. It should be stressed that statistically significant findings across an entire cohort sometimes result from strong trends within a large patient subgroup. We have not established that epithelial events have pathogenetic significance in all patients with SSc-ILD. However, our findings suggest that attempts to deconstruct core pathogenetic mechanisms in the whole cohort of SSc-ILD patients without incorporating epithelial injury may be fatally flawed.

The fact that increased ${ }^{99} \mathrm{~m}$ Tc-DTPA clearance was less predictive of outcome in patients who were treated from presentation is also compatible with an epithelial pathogenetic pathway. In a recent placebo-controlled trial [29], oral cyclophosphamide therapy was associated with a reduction in disease progression. However, the difficulties in controlling for a treatment effect in a large retrospective cohort [14], even with the use of a prospective clinical protocol, should be emphasised. In many cases, therapy is introduced after a period of observation and the choice, timing and duration of treatment necessarily vary widely during follow-up, due to side-effects or disease progression. This problem is partially addressed by separate analyses based on initial management, with the designation of "intention to treat" and "intention to observe" subgroups [14]. In the latter group, it was our policy to introduce treatment only if disease progression was observed and this probably accounts for the stronger linkage between epithelial injury and decline in FVC in this subgroup. Even in a prospective study, the constraints discussed above are likely to hinder the deconstruction of a treatment effect, except in the setting of large placebo-controlled treatment cohorts.

The findings in the present study suggest that attempts to delineate the mathematical components of ${ }^{99 \mathrm{~m}}$ Tc-DTPA clearance do not add to prognostic evaluation in SSc-ILD. Disease progression was linked to increased rapidity of the fast component of clearance, whether evaluated as a discrete variable or as a proportion of overall clearance. However, these relationships were weaker than those between total clearance and outcome. It has been argued that the fast component of clearance might represent clearance in injured lung, with the slow component reflecting clearance in normal lung. The observations of MOGULKOC et al. [8] provide some 
support for this hypothesis, with the fast component of clearance predicting mortality in IPF. However, we were unable to reproduce this finding or to detect any associations between abnormally rapid clearance and disease progression in IPF.

The negative findings in IPF in our study were, at first glance, surprising, as the core pathogenetic pathway is now widely viewed as an epithelial-fibrotic process. However, there are at least two possible explanations for this apparent discrepancy. The usual histopathological pattern in SSc-ILD is nonspecific interstitial pneumonia, in which disease is diffuse and homogenous. By contrast, usual interstitial pneumonia in IPF is heterogeneous, with honeycomb and fibrotic changes interspersed with normal lung. The profusion of fibroblastic foci, and not the extent of fibrosis, is linked to outcome in histological studies of IPF [6, 7], but fibroblastic foci make up only a tiny percentage of abnormal lung, and there is no current method of deconstructing their contribution to overall clearance abnormalities: serum biomarkers that directly reflect focal epithelial events may be more useful in this regard. A second confounding factor is the occurrence of acute exacerbations, which are increasingly recognised in IPF and may profoundly weaken relationships between outcome and measures of epithelial injury at presentation.

However, one intriguing observation emerged from curve deconstruction in IPF: delayed clearance of the slow component was associated with a shorter time to decline in FVC. Thus, in IPF, there are regions of abnormally slow clearance that, when more extensive, denote a poor outcome, a phenomenon that is not seen in SSc-ILD. Although not addressed by our study, this finding may represent the presence of honeycombing in IPF, which is also an adverse prognostic determinant. It is known that honeycomb lung ventilates normally in IPF but is entirely nonperfused, leading to the simulation of pulmonary embolism on ventilationperfusion scanning [30]. Thus, it can be expected that a ${ }^{99 \mathrm{~m}} \mathrm{Tc}-$ DTPA aerosol should deposit normally in honeycomb lung but would not be cleared, accounting for the prognostic significance of delayed slow clearance in the present study.

Some bias exists due to patient selection, with IPF patients less likely to undergo ${ }^{99 \mathrm{~m}}$ Tc-DTPA than SSc-ILD patients as a matter of unit policy, with the existence of a weekly quota of clearance measurements for routine clinical purposes. Another limitation is that the retrospective nature of our analyses, but it should be stressed that all patients underwent a prospective protocol of investigation, except when demand exceeded the routine availability of ${ }^{99 \mathrm{~m}}$ Tc-DTPA clearance measurements. No differences were disclosed when baseline characteristics were compared between the study cohort and patients not undergoing ${ }^{99 \mathrm{~m}}$ Tc-DTPA measurement, either in IPF or SScILD and, thus, it appears unlikely that these biases had a major confounding effect.

In conclusion, our findings indicate that epithelial injury may have a central pathogenetic role in some or all patients with SSc-ILD, independent of disease severity: pathogenetic models in this disease that include no epithelial component may be fatally flawed. By contrast, although IPF pathogenesis is viewed as an epithelial-fibrotic process, outcome is not linked to the extent of epithelial injury, as judged by clearance of ${ }^{99 m}$ Tc-DTPA, suggesting that core pathogenetic events may be more spatially focal in IPF than in SSc-ILD, and may be better captured by biomarkers of disease activity.

\section{SUPPORT STATEMENT}

The project was funded by the UK Raynauds and Sclerodema Association and was supported by the NIHR Respiratory Disease Research Unit at the Royal Brompton and Harefield NHS Foundation Trust and Imperial College London.

\section{STATEMENT OF INTEREST}

A statement of interest for C.P. Denton can be found at www.erj. ersjournals.com/site/misc/statements.xhtml

\section{ACKNOWLEDGEMENTS}

We would like to thank J. Bailey (Dept of Nuclear Medicine, Royal Brompton Hospital, London, UK) for his time and efforts with the collection of DTPA data.

\section{REFERENCES}

1 Wells AU, Hansell DM, Harrison NK, et al. Clearance of inhaled ${ }^{99 m}$ Tc-DTPA predicts the clinical course of fibrosing alveolitis. Eur Respir J 1993; 6: 797-802.

2 Yamane K, Ihn H, Kubo M, et al. Serum levels of KL-6 as a useful marker for evaluating pulmonary fibrosis in patients with systemic sclerosis. J Rheumatol 2000; 27: 930-934.

3 Yanaba K, Hasegawa M, Hamaguchi Y, et al. Longitudinal analysis of serum KL-6 levels in patients with systemic sclerosis: association with the activity of pulmonary fibrosis. Clin Exp Rheumatol 2003; 21: 429-436.

4 Selman M, King TE, Pardo A. Idiopathic pulmonary fibrosis: prevailing and evolving hypotheses about its pathogenesis and implications for therapy. Ann Intern Med 2001; 134: 136-151.

5 Selman M, Pardo A. Role of epithelial cells in idiopathic pulmonary fibrosis: from innocent targets to serial killers. Proc Am Thorac Soc 2006; 3: 364-372.

6 Nicholson AG, Fulford LG, Colby TV, et al. The relationship between individual histologic features and disease progression in idiopathic pulmonary fibrosis. Am J Respir Crit Care Med 2002; 166: 173-177.

7 King TE Jr, Schwarz MI, Brown K, et al. Idiopathic pulmonary fibrosis: relationship between histopathologic features and mortality. Am J Respir Crit Care Med 2001; 164: 1025-1032.

8 Mogulkoc N, Brutsche MH, Bishop PW, et al. Pulmonary ${ }^{99 \mathrm{~m}} \mathrm{Tc}-$ DTPA aerosol clearance and survival in usual interstitial pneumonia (UIP). Thorax 2001; 56: 916-923.

9 Barrowcliffe MP, Jones JG. Solute permeability of the alveolar capillary barrier. Thorax 1987; 42: 1-10.

10 Harrison NK, Glanville AR, Strickland B, et al. Pulmonary involvement in systemic sclerosis: the detection of early changes by thin section CT scan, bronchoalveolar lavage and ${ }^{99 \mathrm{~m}} \mathrm{Tc}-\mathrm{DTPA}$ clearance. Respir Med 1989; 83: 403-414.

11 Antoniou KM, Malagari K, Tzanakis N, et al. Clearance of technetium-99m-DTPA and HRCT findings in the evaluation of patients with idiopathic pulmonary fibrosis. BMC Pulm Med 2006; 6: 4 .

12 Diot E, Giraudeau B, Maillot F, et al. Decrease in DL,CO in systemic sclerosis correlates with acceleration of DTPA clearance. Eur Respir J 1999; 14: 728-729.

13 Latsi PI, du Bois RM, Nicholson AG, et al. Fibrotic idiopathic interstitial pneumonia: the prognostic value of longitudinal functional trends. Am J Respir Crit Care Med 2003; 168: 531-537. 
14 Goh NS, Desai SR, Veeraraghavan S, et al. Interstitial lung disease in systemic sclerosis: a simple staging system. Am J Respir Crit Care Med 2008; 177: 1248-1254.

15 Antoniou KM, Hansell DM, Rubens MB, et al. Idiopathic pulmonary fibrosis: outcome in relation to smoking status. Am J Respir Crit Care Med 2008; 177: 190-194.

16 American Thoracic Society. Idiopathic pulmonary fibrosis: diagnosis and treatment. International consensus statement. American Thoracic Society (ATS), and the European Respiratory Society (ERS). Am J Respir Crit Care Med 2000; 161: 646-664.

17 Wells AU, Desai SR, Rubens MB, et al. Idiopathic pulmonary fibrosis: a composite physiologic index derived from disease extent observed by computed tomography. Am J Respir Crit Care Med 2003; 167: 962-969.

18 Coates G, O'Brodovich H. Measurement of pulmonary epithelial permeability with ${ }^{99 \mathrm{~m}}$ Tc-DTPA aerosol. Semin Nucl Med 1986; 16 : 275-284.

19 Jones JG, Minty BD, Royston D. The physiology of leaky lungs. Br J Anaesth 1982; 54: 705-721.

20 Raghu G, Brown KK, Bradford WZ, et al. A placebo-controlled trial of interferon gamma- $1 \mathrm{~b}$ in patients with idiopathic pulmonary fibrosis. N Engl J Med 2004; 350: 125-133.

21 Kahaleh B. The microvascular endothelium in scleroderma. Rheumatology (Oxford) 2008; 47: Suppl. 5, v14-v15.

22 Harrison NK, Myers AR, Corrin B, et al. Structural features of interstitial lung disease in systemic sclerosis. Am Rev Respir Dis 1991; 144: 706-713.
23 Willis BC, Dubois RM, Borok Z. Epithelial origin of myofibroblasts during fibrosis in the lung. Proc Am Thorac Soc 2006; 3: 377-382.

24 Kapanci Y, Desmouliere A, Pache JC, et al. Cytoskeletal protein modulation in pulmonary alveolar myofibroblasts during idiopathic pulmonary fibrosis. Possible role of transforming growth factor beta and tumour necrosis factor alpha. Am J Respir Crit Care Med 1995; 152: 2163-2169.

25 Khalil N, Greenberg AH. The role of TGF- $\beta$ in pulmonary fibrosis. Ciba Found Symp 1991; 157: 194-207.

26 Antoniades HN, Bravo MA, Avila RE, et al. Platelet-derived growth factor in idiopathic pulmonary fibrosis. J Clin Invest 1990; 86: 1055-1064.

27 Nash JR, McLaughlin PJ, Butcher D, et al. Expression of tumour necrosis factor- $\alpha$ in cryptogenic fibrosing alveolitis. Histopathology 1993; 22: 343-347.

28 Hoyles RK, Khan K, Shiwen X, et al. Fibroblast-specific perturbation of transforming growth factor beta signaling provides insight into potential pathogenic mechanisms of scleroderma-associated lung fibrosis: exaggerated response to alveolar epithelial injury in a novel mouse model. Arthritis Rheum 2008; 58: 1175-1188.

29 Tashkin DP, Elashoff R, Clements PJ, et al. Cyclophosphamide versus placebo in scleroderma lung disease. N Engl J Med 2006; 354 : 2655-2666.

30 Strickland NH, Hughes JM, Hart DA, et al. Cause of regional ventilation-perfusion mismatching in patients with idiopathic pulmonary fibrosis: a combined CT and scintigraphic study. Am J Roentgenol 1993; 161: 719-725. 